\title{
Racial and geographic variation in coronary heart disease mortality trends
}

Richard F Gillum ${ }^{1,2^{*}}$, Alem Mehari ${ }^{1}$, Bryan Curry ${ }^{1}$ and Thomas O Obisesan ${ }^{1,2^{*}}$

\begin{abstract}
Background: Magnitudes, geographic and racial variation in trends in coronary heart disease (CHD) mortality within the US require updating for health services and health disparities research. Therefore the aim of this study is to present data on these trends through 2007.

Methods: Data for CHD were analyzed using the US mortality files for 1999-2007 obtained from the US Centers for Disease Control and Prevention. Age-adjusted annual death rates were computed for non-Hispanic African Americans (AA) and European Americans (EA) aged 35-84 years. The direct method was used to standardize rates by age, using the 2000 US standard population. Joinpoint regression models were used to evaluate trends, expressed as annual percent change (APC).
\end{abstract}

Results: For both AA men and women the magnitude in CHD mortality is higher compared to EA men and women, respectively. Between 1999 and 2007 the rate declined both in AA and in EA of both sexes in every geographic division; however, relative declines varied. For example, among men, relative average annual declines ranged from $3.2 \%$ to $4.7 \%$ in $\mathrm{AA}$ and from $4.4 \%$ to $5.5 \%$ in EA among geographic divisions. In women, rates declined more in later years of the decade and in women over 54 years. In 2007, age-adjusted death rate per 100,000 for CHD ranged from 93 in EA women in New England to 345 in AA men in the East North Central division. In EA, areas near the Ohio and lower Mississippi Rivers had above average rates. Disparities in trends by urbanization level were also found. For AA in the East North Central division, the APC was similar in large central metro (-4.2), large fringe metro (-4.3), medium metro urbanization strata $(-4.4)$, and small metro (-3.9). APC was somewhat higher in the micropolitan/non-metro $(-5.3)$, and especially the non-core/non-metro (-6.5). For EA in the East South Central division, the APC was higher in large central metro (-5.3), large fringe metro (-4.3) and medium metro urbanization strata $(-5.1)$ than in small metro $(-3.8)$, micropolitan/non-metro $(-4.0)$, and non-core/non-metro (-3.3) urbanization strata.

Conclusions: Between 1999 and 2007, the level and rate of decline in CHD mortality displayed persistent disparities. Declines were greater in EA than AA racial groups. Rates were greater in the Ohio and Mississippi River than other geographic regions.

Keywords: Coronary heart disease, African Americans, Mortality

\footnotetext{
* Correspondence: rfg2.howard.edu@gmail.com; tobisesan@Howard.edu

'Department of Medicine, Howard University College of Medicine,

Washington, DC, USA

2Division of Geriatrics, Howard University Hospital, Towers Bldg. 2041 Georgia

Ave, NW, Washington DC 20060, USA
}

\section{Biomed Central}




\section{Background}

In 2009, despite a long-term decline in death rates, coronary heart disease (CHD) was the leading cause of death in the US [1-4]. Although reductions in gender disparities in CHD mortality have been noted [5], disparities in CHD mortality based on race/ethnicity have remained largely unchanged [6], and disparities in the morbidity of CHD appear to be increasing [7]. Many publications have examined the decline in CHD mortality in the US prior to 2000, but relatively few have detailed both racial and geographic variation $[1,8-20]$. The Healthy People 2010 Initiative set the goals of reducing the CHD mortality rate by 20 percent between 2000 and 2010 and reducing disparities in CHD mortality. The Healthy People 2010 objectives of reducing death rates to 162 deaths per 100,000 populations for CHD were met in 2004. However, despite the overall decrease in CHD death rates, the target death rates were not met for two subpopulations: blacks and men [3]. Two out of the four overarching goals of Healthy People 2020 are: 1) achieve health equity, eliminate disparities, and improve the health of all groups; and; 2) Examining and monitoring the distribution of death rates provides the requisite information for focusing on the groups most in need of early intervention to eliminate preventable disease, disability, and premature death and to improve the health of all groups [4]. Magnitudes, geographic and racial variation in trends in CHD mortality within the US require frequent updating for health services and health disparities research.

Accordingly, this report presents CHD mortality trends between 1999-2007 among non-Hispanic blacks and whites aged 35-84 years and geographic and racial variation in CHD mortality trends.

\section{Materials and methods}

Deaths in 1999-2007 with coronary heart disease (International Classification of Disease $10^{\text {th }}$ revision [ICD-10] codes I20-I25 (1999-2007) as underlying cause were enumerated [20]. Data available for public use from National Vital Statistics System (NVSS), files provided the underlying cause of death and demographic data on decedents [20]. The underlying cause of death is the disease (or injury) that initiated the sequence of events leading directly to death. For non-Hispanic African Americans (AA) and European Americans (EA), age-adjusted death rates per 100,000 using the 2000 US standard population with 95\% confidence intervals $(\mathrm{CI})$ were computed for persons aged 35-84 years using standard methods [20]. Following policy of the NVSS, years and areas were combined to avoid producing death rates considered unreliable, i.e. when the death count is less than 20. Variation by sex and race in annual percentage change (APC) between 1999 and 2007 were assessed using JoinPoint software [21]. Joinpoint is statistical software for the analysis of trends using joinpoint models, that is, models where several different lines are connected together at the "joinpoints". This enables the user to test that an apparent change in trend is statistically significant. The tests of significance use a Monte Carlo Permutation method. Numbers of U.S. residents for the period 1999-2007 were obtained from the U.S. Bureau of the Census and used to calculate death rates per 100,000 populations. Bridged-race Census estimates were used as denominators for groups defined by race and Hispanic origin. Urbanization levels and Census divisions are defined in Additional file 1: Table S1 [22].

\section{Results}

In 2007 at ages 35-84, US age-adjusted CHD death rates per 100,000 (95\% CI) were as follows: women EA 111 (110-112), AA 171 (168-174); men EA 238 (237-239), AA 312 (307-316). Examination of annual percent change (APC) and US age-adjusted CHD death rates between 1999 and 2007 for women and men at ages 35-84 yrs revealed that rates declined for AA and EA men at ages 35-84, and in each age subgroup (Table 1). In both EA and AA women, the rates declined more rapidly in later years compared to the earlier years of the decade. In women, APC was greater above age 54 than at 35-54 years, which the APC for EA women barely different from 0 (Table 1).

Table 2 shows states with in order of decreasing age adjusted CHD mortality rates for AA in 2005-2007, while Additional file 2: Tables S2 and Additional file 3: Table S3 show state rankings for EA. Relative variation was greatest in AA men and least in AA women. For the entire period 1999-2007, Figures 1 and 2 show that high death rates for EA men and women tended to cluster around the Ohio and lower Mississippi Rivers and low rates in Rocky Mountains (Additional file 2: Tables S2 and Additional file 3: Table S3). Clustering of high rates for both AA men and women was seen in the East North Central, West South Central states, Middle Atlantic States and California and low rates in South Atlantic and East South Central states (see Additional file 4: Figures S1 and Additional file 5: Figure S2. The ratio of the 90th to the 10th percentile of rates in states for EA and AA combined was 1.67 in 1999 and 1.76 in 2007 (1.61 and 1.67 resp. in EA only).

In 1999-2007 rates declined in all the nine US Census divisions (Table 3). Relative declines tended to be greatest in the New England, Mountain and Pacific divisions. Variation in rate of decline was greater in AA than in EA. The greatest relative declines were in AA women in the East South Central and New England divisions and the smallest relative declines occurred in AA males in the Pacific, MidAtlantic and North Central divisions.

For AA within the Mid-Atlantic, East North Central and Pacific divisions where over 6 million AA aged 35-84 resided, age-adjusted rates for 1999-2007 combined were highest in large, central metropolitan (LCM) areas, e.g. Mid- 
Table 1 Annual percent change age-adjusted rates of mortality from coronary heart disease per 100,000 by race in non-Hispanic women and men aged 35-84+ years: United States, 1999-2007

\begin{tabular}{|c|c|c|c|c|c|}
\hline Characterstic & Year & AA women & EA women & AA men & EA men \\
\hline APC(CI)35-84Y & 1999-2007 & - & - & $-4.0(-4.6,-3.4)$ & $-4.8(-5.1,-4.5)$ \\
\hline Rate & 1999 & - & - & 329 & 346 \\
\hline Rate & 2007 & - & - & 246 & 240 \\
\hline APC(Cl)35-84Y & 2003-2007 & - & $-6.2(-7.2,-5.1)$ & - & - \\
\hline Rate & 2003 & - & 143 & - & - \\
\hline Rate & 2007 & - & 111 & - & - \\
\hline APC(Cl)35-84Y & 1999-2003 & - & $-4.5(-5.5,-3.5)$ & - & - \\
\hline Rate & 1999 & - & 172 & - & - \\
\hline Rate & 2003 & - & 143 & - & - \\
\hline APC(Cl)35-84Y & $2002-2007$ & $-6.3(-7.0,-5.6)$ & - & - & - \\
\hline Rate & 2002 & 237 & - & - & - \\
\hline Rate & 2007 & 171 & - & - & - \\
\hline APC(CI)35-84Y & 1999-2002 & $-3.7(-5.2,-2.1)$ & - & - & - \\
\hline Rate & 1999 & 265 & - & - & - \\
\hline Rate & 2002 & 237 & - & - & - \\
\hline APC(CI) 35-54Y & 1999-2007 & $-3.2(-3.9,-2.6)$ & $-0.8(-1.4,-0.1)$ & $-2.6(-3.2 .-2.0)$ & $-2.1(-2.4,-1.7)$ \\
\hline APC(CI) 55-84Y & 1999-2007 & $-5.7(-6.3,-5.1)$ & $-5.7(-6.2,-5.2)$ & $-4.3(-4.9,-3.6)$ & $-5.2(-5.5,-4.9)$ \\
\hline $\mathrm{APC}(\mathrm{Cl})>84 \mathrm{Y}$ & 1999-2007 & $-5.2(-6.2,-4.2)$ & $-5.9(-6.5,-5.2)$ & $-6.1(-6.8,-5.5)$ & $-5.2(-5.7,-4.8)$ \\
\hline
\end{tabular}

AA, African American; EA, European American; APC, annual percent change; $\mathrm{Cl}, 95 \%$ confidence interval; $\mathrm{Y}=$ ages in years.

Atlantic LCM 439 (95\% CI 436-443) versus large fringe metro (LFM) 372 (366-379). In South Atlantic and East South Central divisions, rates showed a similar pattern but at somewhat lower levels e.g. South Atlantic LCM 369 (365-373) versus LFM 311 (307-315). In New England, West North Central and West South Central division's rates were highest in the non-core (non-metropolitan) areas.

For EA within the New England, Mid-Atlantic, East North Central and Pacific divisions, age-adjusted rates for 1999-2007 combined were highest in LCM areas, e.g. Mid-Atlantic LCM 276 (95\% CI 274-278) versus LFM 209 (208-210). In West North Central, South Atlantic, East South Central and West South Central divisions rates were highest in the non-core(non-metropolitan) divisions, e.g. East South Central non-core(non-metropolitan) division 281 (278-284) versus LCM 212 (209-215).

Trends were examined by urbanization level in the East North Central division for AA and the East South Central division for EA, i.e. the divisions with highest rates. For AA in the East North Central division, the APC was similar in large central metro (-4.2), large fringe metro (-4.3), medium metro urbanization strata (-4.4), and small metro (-3.9). The somewhat higher APC in the micropolitan/ non-metro (-5.3), and especially the non-core/non-metro (-6.5) urbanization strata must be viewed with caution due to much smaller annual numbers of deaths.

For EA in the East South Central division, the APC was higher in large central metro (-5.3), large fringe metro
$(-4.3)$ and medium metro urbanization strata $(-5.1)$ than in small metro (-3.8), micropolitan/non-metro (-4.0), and non-core/non-metro (-3.3) urbanization strata.

\section{Discussion}

The long-term decline in CHD mortality rates has continued during the first decade of the new century for major demographic subgroups. However, the historic racial disparities and substantial geographic disparities persist. Hence, the Healthy People 2010 goal of a 20\% decline between 2000 and 2010 likely will be met or exceeded (2000-2007 all ages decline 33\%), but little or no progress towards the goal of elimination of racial or geographic relative disparities during the decade is evident.

Many studies have documented the magnitude and the important contributions of both improvements in health care and improvements in population risk factor levels in reducing CHD incidence rates and reducing case fatality rates for acute myocardial infarction and long-term survival with $C H D[1,3-5,8,13-18]$. Combined US racial and geographic variation in levels and trends in CHD mortality or morbidity has been the subject of a smaller number of studies [1,8-19]. The causes of the large racial and geographic variation in CHD mortality have yet to be fully identified [23-30]. Disparities in access to and quality of therapeutic and preventive health care and socioeconomic and cultural variation in lifestyles and risk factor levels are thought to play important roles [29,30]. For example, 
Table 2 States* with the highest and lowest age-adjusted coronary heart disease mortality rate per 100,000 by sex and race in non-Hispanic African Americans aged 35-84 years: United States, 2005-2007

\begin{tabular}{|c|c|c|}
\hline & Women & Men \\
\hline & Michigan & Michigan \\
\hline & New York & Oklahoma \\
\hline & Arkansas & Arkansas \\
\hline & Oklahoma & California \\
\hline & California & Tennessee \\
\hline & Tennessee & New York \\
\hline & Missouri & Missouri \\
\hline & Illinois & Pennsylvania \\
\hline & Ohio & Illinois \\
\hline & Texas & Mississippi \\
\hline & Maryland & Louisiana \\
\hline & Mississippi & Indiana \\
\hline & Louisiana & Texas \\
\hline & Kentucky & Ohio \\
\hline & Arizona & West Virginia \\
\hline & Pennsylvania & Kentucky \\
\hline & New Jersey & Maryland \\
\hline & Florida & North Carolina \\
\hline & Indiana & New Jersey \\
\hline & Delaware & South Carolina \\
\hline & North Carolina & Delaware \\
\hline & Kansas & Florida \\
\hline & South Carolina & Arizona \\
\hline & Virginia & Wisconsin \\
\hline & Nevada & Virginia \\
\hline & Alabama & Kansas \\
\hline & Washington & Washington \\
\hline & Georgia & Alabama \\
\hline & Connecticut & Connecticut \\
\hline & Colorado & Nevada \\
\hline & Massachusetts & Georgia \\
\hline & Connecticut & Massachusetts \\
\hline & & Colorado \\
\hline & & Minnesota \\
\hline Ratio $^{+}$ & 2.1 & 3.1 \\
\hline
\end{tabular}

*Excludes states with 99 or fewer deaths in 2005-2007 for given group. ${ }^{+}$Ratio of rate of state in first row to state in last row.

persistent racial disparities in utilization of revascularization produces have been well documented [31,32].

Early investigators noted high stroke mortality rates among Americans in the southeastern coastal states. By late 1980s, this "stroke belt" had dissipated in the south eastern coastal areas and shifted to the Midwest regions of the Mississippi and Ohio River valleys [8]. CHD mortality has shown a similar westward shift to the so called coronary valley [9]. The increased cardiovascular disease (CVD) mortality among blacks has been mainly confined to the southern states (Mississippi River valley). Although changes in regional profile of risk factors, local environment, and migration pattern may have played a role, recent economic shifts in these areas may be the principal reason for changes in disease rates [8]. Whereas southeastern coastal areas have undergone considerable economic development, the more westward regions have not kept pace [8]. In Mississippi, one of the most economically and educationally disadvantaged US states, CVD mortality has risen among African Americans over the past two decades while among whites there has been a decline [10]. Similar patterns are also observed in the Northeast and the Midwest $[11,12]$.

Our understanding of the existence in race/ethnic variations in CHD disease burden comes from observed differences in their rates and risk factors between countries. For example, in the Seven Countries Study, [33], low CHD rates were observed in Japan and the Mediterranean countries, and high CHD rates in Finland and the US. These differences were in large part explained by differences in risk factors, but several factors may contribute to these observed differences, such as diverse demographic profiles, environmental factors, and genetic factors differences. These variations are perhaps best illustrated by the knowledge gained from studies in migrants. The Ni-Hon-San study of Japanese migrants revealed how blood cholesterol levels and CHD rates rose from relatively low levels among those in Japan, to intermediate levels in Honolulu, and to high levels in San Francisco [34].

Comparison of Afro-Caribbeans, South Asians, and Europeans in the UK indicate marked differences in central obesity, glucose intolerance, hyperinsulinemia, and related dyslipidemia, despite similar blood pressure, body mass index, and total plasma cholesterol [35]. In Canada, there are marked differences between different ethnic groups in the prevalence and death rates from CHD, with the highest rates being among those of European and South Asian origin, but lowest among those of Chinese origin [36], suggesting that the propensity to CHD may vary in different ethnic groups.

Differences in the age-adjusted death rates vary widely between European populations. Data from the World Health Organization (WHO) indicate that the cardiovascular disease mortality rate is 6-fold higher among men and women in the Russian Federation compared with people in France [37]. In 1996, the age-standardized mortality rates for coronary heart disease (CHD) among males in the Russian Federation was 390/100 000 compared with 60/100 000 among males in France [37]. 


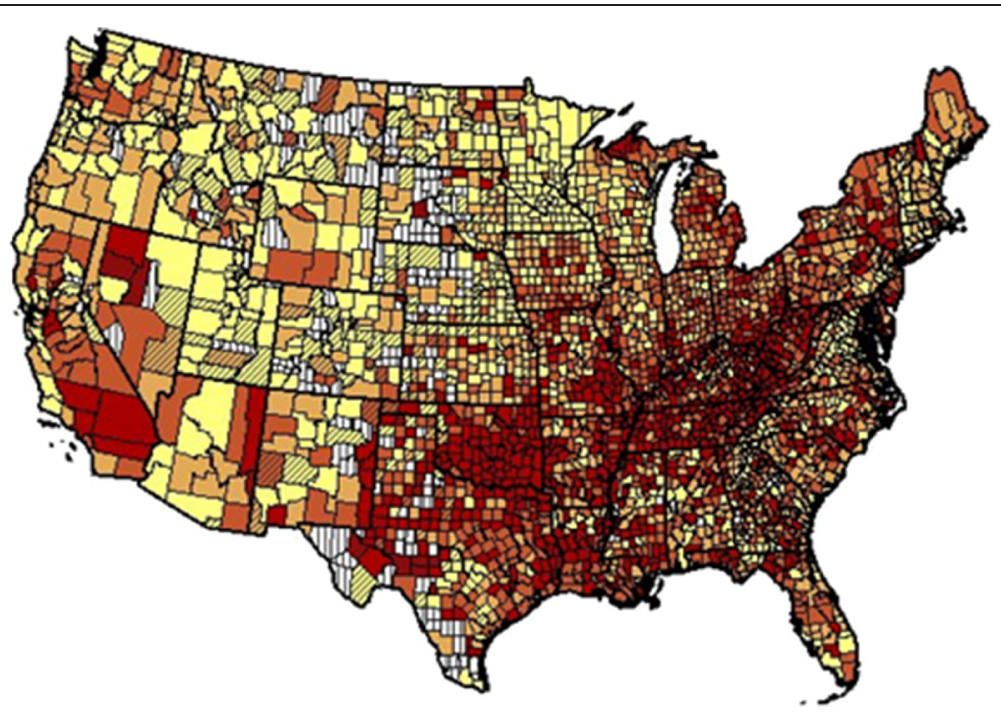

Figure 1 Age-adjusted rate per 100,000 resident population of death from coronary heart disease by county for European American women aged 35-84 years: United States, 1999-2007. Yellow, 34.7-114.5; light orange 114.6-142.0; dark orange 142.1-172.2; red 172.4-407.2; white vertical bars, suppressed due to small number of deaths; color diagonal bars, unreliable value.

Although the CVD mortality rates are much lower among women compared with men, similar variations among women between countries also exist. Eastern European countries such as the Ukraine, the Russian Federation, Hungary, and the Czech Republic have among the highest and increasing CVD rates in the world, which is in marked contrast to most economically stable European countries where declines in CVD mortality rates have been experienced over the past 30 years [38]. CVD among
European populations is mainly attributable to classical risk factors, namely diets high in saturated fats, elevated serum cholesterol and blood pressure (BP), diabetes, and smoking [39].

Strengths of this study include focus on the years 1999-2007 when only ICD-10 was in use, which precluded bias due to changes in ICD version [20]. Unlike some previous studies, analyses were restricted to nonHispanics to avoid bias arising from misclassification of

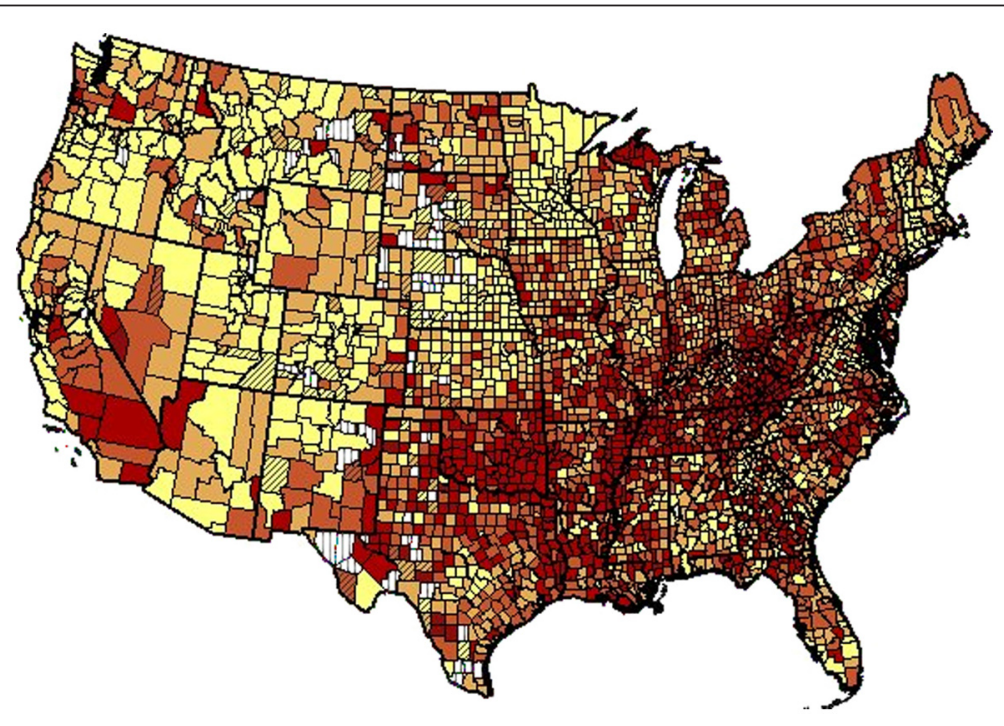

Figure 2 Age-adjusted rate of death per 100,000 population from coronary heart disease by county for European American men aged 35-84 years: United States, 1999-2007. Yellow 56.8-252.0; light orange 252.1-300.4; dark orange 300.6-356.3; red 356.4-743.4; white vertical bars, suppressed due to small number of deaths; color diagonal bars, unreliable value. 
Table 3 Annual percent change and selected age-adjusted rates of mortality from coronary heart disease per 100,000 by Census division, sex and race in non-Hispanics aged 35-84 years: United States, 1999-2007

\begin{tabular}{|c|c|c|c|c|c|}
\hline Division & & AA Women & EA Women & AA Men & EA Men \\
\hline \multicolumn{6}{|c|}{ New England } \\
\hline APC & 1999-2007 & -6.2 & -5.9 & -4.7 & -5.5 \\
\hline Rate & 1999 & 176 & 144 & 329 & 306 \\
\hline Rate & 2007 & 111 & 93 & 230 & 202 \\
\hline \multicolumn{6}{|c|}{ Mid Atlantic } \\
\hline APC & 1999-2007 & -3.9 & -5.4 & -3.5 & -4.9 \\
\hline Rate & 1999 & 284 & 197 & 452 & 379 \\
\hline Rate & 2007 & 203 & 124 & 345 & 259 \\
\hline \multicolumn{6}{|c|}{ E. N. Central } \\
\hline APC & 1999-2007 & -5.2 & -5.6 & -3.6 & -5.0 \\
\hline Rate & 1999 & 285 & 181 & 487 & 374 \\
\hline Rate & 2007 & 187 & 113 & 352 & 248 \\
\hline \multicolumn{6}{|c|}{ W. N. Central } \\
\hline APC & 1999-2007 & -4.9 & -5.3 & -3.5 & -4.8 \\
\hline Rate & 1999 & 274 & 151 & 428 & 324 \\
\hline Rate & 2007 & 175 & 95 & 319 & 217 \\
\hline S. Atlantic & AAPC & & & & \\
\hline APC & 1999-2007 & -6.1 & -5.8 & -4.7 & -5.1 \\
\hline Rate & 1999 & 238 & 165 & 395 & 344 \\
\hline Rate & 2007 & 148 & 102 & 273 & 227 \\
\hline \multicolumn{6}{|c|}{ E. S. Central } \\
\hline APC & 1999-2007 & -6.4 & -4.5 & -4.1 & -4.4 \\
\hline Rate & 1999 & 266 & 189 & 434 & 398 \\
\hline Rate & 2007 & 162 & 132 & 309 & 276 \\
\hline \multicolumn{6}{|c|}{ W. S. Central } \\
\hline APC & 1999-2007 & -5.8 & -4.6 & -4.1 & -4.5 \\
\hline Rate & 1999 & 276 & 189 & 461 & 385 \\
\hline Rate & 2007 & 172 & 130 & 332 & 267 \\
\hline \multicolumn{6}{|l|}{ Mountain } \\
\hline APC & 1999-2007 & -5.7 & -4.8 & -5.6 & -4.6 \\
\hline Rate & 1999 & 229 & 137 & 332 & 295 \\
\hline Rate & 2007 & 140 & 89 & 193 & 196 \\
\hline \multicolumn{6}{|l|}{ Pacific } \\
\hline APC & 1999-2007 & -5.3 & -5.5 & -3.2 & -4.8 \\
\hline Rate & 1999 & 308 & 172 & 471 & 339 \\
\hline Rate & 2007 & 195 & 107 & 346 & 224 \\
\hline
\end{tabular}

AA, African American; EA, European American.

Hispanic ethnicity on death certificates. However, given the lack of information on racial and regional variation in validity of CHD diagnoses from death certificates, bias due to diagnostic misclassification cannot be excluded as a possible contributor to variation [40]. The validity of using age-adjusted rates for ages 65 yrs and over may be a problem as rates are driven by oldest age groups for CHD where diagnosis accuracy may be questionable. Results of trends in age-adjusted rates within three age bands were performed for this reason. Ecologic analyses of clinical information for cases or data on access to care or risk factor levels, socioeconomic and health service variables in different geographic areas could have confounded or mediated some of the results but analysis of those variables was beyond the scope of this study. More such efforts are needed to explain observed racial and geographic patterns. Continued monitoring of racial and geographic disparities will be of especial importance in an era of health care system changes.

\section{Conclusions}

Between 1999 and 2007, the level and rate of decline in CHD mortality displayed persistent disparities. Rates were greater in the Ohio and Mississippi River than other geographic regions. Declines were greater in EA than AA racial groups.

\section{Additional files}

Additional file 1: Table S1. Classification rules used to assign counties to the six urbanization levels of the 2006 NCHS Urban-Rural Classification.

Additional file 2: Table S2. Age-adjusted coronary heart disease mortality rate per 100,000 by state in non-Hispanic European American women aged 35-84 years: United States, 2005-2007.

Additional file 3: Table S3. Age-adjusted coronary heart disease mortality rate per 100,000 by state in non-Hispanic European American men aged 35-84 years: United States, 2005-2007.

Additional file 4: Figure S1. Age-adjusted rate per 100,000 resident population of death from coronary heart disease by state for African American women aged 35-84 years: United States, 2005-2007. Legend: yellow 63-139, light orange $>141-170$, dark orange $>173-188$, red $>199-259$.

Additional file 5: Figure S2. Age-adjusted rate of death per 100,000 population from coronary heart disease by state for African American men aged 35-84 years: United States, 2005-2007. Legend: yellow 88-238, light orange $>243-304$, dark orange $>320-356$, red $>360-583$.

\section{Competing interests}

The authors declare that they have no competing interests.

\section{Authors' contributions}

RG has been involved in conception of the study, analysis and interpretation of data and drafting the manuscript and revising it critically for important intellectual content. AM and TO have participated in analysis, interpretation of data and revising the manuscript critically for important intellectual content. BC participated in reviewing the content of the manuscript. All authors read and approved the final manuscript.

\section{Funding}

Dr. Obisesan was supported by career development award \# AG00980, research award \#RO1-AG031517 from the National Institute on Aging and research award \#1UL1RR03197501 from the National Center for Research Resources. 
Received: 13 January 2012 Accepted: 27 April 2012

Published: 6 June 2012

\section{References}

1. Roger VL, Go AS, Lloyd-Jones DM, Adams RJ, Berry JD, Brown TM, Carnethon MR, Dai S, de Simone G, Ford ES, Fox CS, Fullerton HJ, Gillespie C, Greenlund KJ, Hailpern SM, Heit JA, Ho PM, Howard VJ, Kissela BM, Kittner SJ, Lackland DT, Lichtman JH, Lisabeth LD, Makuc DM, Marcus GM, Marelli A, Matchar DB, McDermott MM, Meigs JB, Moy CS, et al: Heart disease and stroke statistics-2011 update: a report from the American Heart Association. Circulation 2011, 123:e18-e209.

2. Kochanek KD, Xu JQ, Murphy SL, Miniño AM, Kung HC: Deaths: Preliminary Data for 2009. National Vital Statistics Reports. vol 59 no 4th edition. Hyattsville: National Center for Health Statistics; 2011.

3. Capewell S, Ford ES, Croft JB, Critchley JA, Greenlund KJ, Labarthe DR: Cardiovascular risk factor trends and potential for reducing coronary heart disease mortality in the United States of America. Bull World Health Organ 2010, 88:120-130.

4. US Department of Health and Human Services: Healthy People 2020.Accessed at http://www.healthypeople.gov/2020/default.aspx on Mar 14, 2012 10:53:42 AM.

5. Pearcy JN, Keppel KG: "A summary measure of health disparity." Public Health Rep 2002, 117(3):273-280.

6. O'Connell L, Brown SL: Do nonprofit HMOs eliminate racial disparities in cardiac care. J Health Care Finance 2003, 30(2):84-94

7. Davis S, Liu Y, Gibbons G: Disparities in trends of hospitalization for potentially preventable chronic conditions among African Americans during the 1990s: implications and benchmarks. Am J Public Health 2003, 93(3):447-455.

8. Casper ML, Wing S, Knoless M, Pollard RA: The shifting stroke belt: changes in the geographic pattern of stroke mortality in the United States, 1962 to 1988. Stroke 1995, 26(5):755-760.

9. Casper M, Wing S, Strogatz D: Variation in the magnitude of black-white differences in stroke mortality by community occupational structure. J Epidemiol Community Health 1991, 45(4):302-306.

10. Jones DW, Sempos CT, Thom TJ, Harrington AM, Taylor HA Jr, Fletcher BW Mehrotra BD, Wyatt SB, Davis CE: Rising levels of cardiovascular mortality in Mississippi, 1979-1995. Am J Med Sci 2000, 319(3):131-137.

11. Geronimus AT, Bound J, Waidmann TA, Hillemeier MM, Burns PB: Excess mortality among blacks and whites in the United States. N Engl J Med 1996, 335(21):1552-1558.

12. Fang J, Madhavan S, Alderman MH: The association between birthplace and mortality from cardiovascular causes among black and white residents of New York City. N Engl J Med 1996, 335(21):1545-1551.

13. Gerber $Y$, Jacobsen SJ, Frye RL, Weston SA, Killian JM, Roger VL: Secular trends in deaths from cardiovascular diseases: a 25-year community study. Circulation 2006, 113:2285-2292.

14. Ford ES, Capewell S: Coronary heart disease mortality among young adults in the U.S. from 1980 through 2002: concealed leveling of mortality rates. J Am Coll Cardiol 2007, 50:2128-2132.

15. Pickle LW, Gillum RF: Geographic variation in cardiovascular disease mortality in US blacks and whites. J Natl Med Assoc 1999, 91:545-556.

16. Gillum RF, Mussolino ME, Madans JH: Coronary heart disease incidence and survival in African-American women and men. The NHANES I Epidemiologic Follow-up Study. Ann Intern Med 1997, 127(2):111-118.

17. Keenan NL, Shaw KM: Centers for Disease Control and Prevention (CDC) Coronary heart disease and stroke deaths - United States, 2006. MMWR Surveill Summ. 2011, 14(60):62-66. 2011 Jan.

18. Centers for Disease Control and Prevention (CDC): Coronary heart disease mortality trends among whites and blacks-Appalachia and United States, 1980-1993. MMWR Morb Mortal Wkly Rep 1998, 47:1005-8. 1015.

19. Centers for Disease Control and Prevention (CDC): State-specific mortality from sudden cardiac death-United States, 1999. MMWR Morb Mortal Wkly Rep 2002, 51(6):123-126.

20. Centers for Disease Control and Prevention, National Center for Health Statistics: Multiple cause of death file 2005-2006. CDC WONDER on-line database, compiled from multiple cause of death file 2005-2006 series 20 no. 21, 2009. Accessed at http://wonder.cdc.gov/mcd-icd10.html on Nov 23, 2010 1:53:42 PM.

21. National Cancer Institute: JointPoint regression program. Accessed at (http:// surveillance.cancer.gov/joinpoint/example.html) Nov 22, 2011, 9:22 AM.

22. Ingram DD, Franco S: 2006 NCHS urban-rural classification scheme for counties. 2006. http://wonder.cdc.gov/wonder/help/CMF/Urbanization-Methodology. html (last accessed 12/20/2010).
23. Peterson ED, Shah BR, Parsons L, Pollack CV Jr, French WJ, Canto JG, Gibson CM, Rogers WJ: Trends in quality of care for patients with acute myocardial infarction in the National Registry of Myocardial Infarction from 1990 to 2006. Am Heart J 2008, 156:1045-1055.

24. Setoguchi S, Glynn RJ, Avorn J, Mittleman MA, Levin R, Winkelmayer WC: Improvements in long-term mortality after myocardial infarction and increased use of cardiovascular drugs after discharge: a 10-year trend analysis. J Am Coll Cardiol 2008, 51:1247-1254.

25. Holmes JS, Kozak LJ, Owings MF: Use and in-hospital mortality associated with two cardiac procedures, by sex and age: national trends, 1990-2004. Health Aff (Millwood). 2007, 26:169-177.

26. Nemetz PN, Roger VL, Ransom JE, Bailey KR, Edwards WD, Leibson CL: Recent trends in the prevalence of coronary disease: a population-based autopsy study of nonnatural deaths. Arch Intern Med 2008, 168:264-270.

27. Wang H, Steffen LM, Jacobs DR, Zhou X, Blackburn H, Berger AK, Filion KB, Luepker RV: Trends in cardiovascular risk factor levels in the Minnesota Heart Survey (1980-2002) as compared with the National Health and Nutrition Examination Survey (1976-2002): A partial explanation for Minnesota's low cardiovascular disease mortality? Am J Epidemiol 2011, 173:526-538.

28. Ford ES, Ajani UA, Croft JB, Critchley JA, Labarthe DR, Kottke TE, Giles WH, Capewell S: Explaining the decrease in U.S. deaths from coronary disease, 1980-2000. N Engl J Med 2007, 356(23):2388-2398.

29. Glasser SP, Cushman M, Prineas R, Kleindorfer D, Prince V, You Z, et al: Does differential prophylactic aspirin use contribute to racial and geographic disparities in stroke and coronary heart disease (CHD)? Prev Med 2008, 47:161-166.

30. Ezzati M, Oza S, Danaei G, Murray CJ: Trends and cardiovascular mortality effects of state-level blood pressure and uncontrolled hypertension in the United States. Circulation 2008, 117(7):905-914.

31. Brown CP, Ross L, Lopez I, Thornton A, Kiros GE: Disparities in the receipt of cardiac revascularization procedures between blacks and whites: an analysis of secular trends. Ethn Dis 2008, 18(2 Suppl 2):S2-112-7. 17.

32. Gillum RF: Coronary revascularization in older women and men in the United States: trends in ethnic differences. Am Heart J 2004, 147:418-424.

33. Menotti A, Keys A, Kromhout D, Blackburn H, Aravanis C, Bloemberg B, Buzina R, Dontas A, Fidanza F, Giampaoli S, Karvonen M, Lanti M, Mohacek I, Nedeljkovic S, Nissinen A, Pekkanen J, Punsar S, Seccareccia F, Toshima H: Inter-cohort differences in coronary heart disease mortality in the 25-year follow-up of the seven countries study. Eur J Epidemio/ 1993, 9(5):527-536

34. Kagan A, Harris BR, Winkelstein W Jr, Johnson KG, Kato H, Syme SL, Rhoads GG, Gay ML, Nichaman MZ, Hamilton HB, Tillotson J: Epidemiologic studies of coronary heart disease and stroke in Japanese men living in Japan, Hawaii and California: demographic, physical, dietary and biochemical characteristics. J Chronic Dis 1974, 27(7-8):345-364.

35. McKeigue PM, Shah B, Marmot MG: Relation of central obesity and insulin resistance with high diabetes prevalence and cardiovascular risk in South Asians. Lancet 1991, 337(8738):382-386.

36. Anand SS, Yusuf S, Vuksan V, Devanesen S, Teo KK, Montague PA, Kelemen L, Yi C, Lonn E, Gerstein H, Hegele RA, McQueen M: Differences in risk factors, atherosclerosis, and cardiovascular disease between ethnic groups in Canada: the Study of Health Assessment and Risk in Ethnic groups (SHARE). Lancet 2000, 356(9226):279-284.

37. 1997-1999 World Health Statistics Annual. Geneva: World Health Organization; 2000. Available at: http://www- nt.who.int/whosis/statistics/ menu.cfm?path=statistics, whsa\&language=english. Accessed March 9, 2012.

38. Artaud-Wild SM, Connor SL, Sexton G, Connor WE: Differences in coronary mortality can be explained by differences in cholesterol and saturated fat intakes in 40 countries but not in France and Finland: a paradox. Circulation 1993, 88:2771-2779.

39. Yusuf S, Reddy S, Ônpuu S, Anand S: Global burden of cardiovascular diseases. Circulation 2001, 104(23):2855-2864.

40. Ives DG, Samuel P, Psaty BM, Kuller LH: Agreement between nosologist and cardiovascular health study review of deaths: implications of coding differences. J Am Geriatr Soc 2009, 57:133-139.

doi:10.1186/1471-2458-12-410

Cite this article as: Gillum et al:: Racial and geographic variation in coronary heart disease mortality trends. BMC Public Health 2012 12:410. 